\title{
Risk Factors for Cervical Cancer and CIN3 in Jewish Women in Israel - Two Case Control Studies
}

\author{
Ravit Bassal ${ }^{1,2 *}$, Eduardo Schejter ${ }^{3}$, Rachel Bachar ${ }^{3}$, Tamar Perri ${ }^{4}$, Jacob \\ Korach $^{4}$, Ariella Jakobson-Setton ${ }^{4}$, Liat Hogen Ben-David ${ }^{4}$, Daniel Cohen ${ }^{1,5}$, \\ Lital Keinan-Boker ${ }^{2,6}$
}

\begin{abstract}
Purpose: The aim of the study was to identify risk and protective factors/markers for cervical cancer and cervical intraepithelial neoplasia 3 (CIN3) in Israeli Jewish women in order to settle the discrepancy of low incidence rate of cervical cancer and relatively high incidence rate of CIN3. Materials and Methods: We conducted two case control studies, which examined the association between potential risk and protective factors/markers for cervical cancer or CIN3 using self administered detailed questionnaires. Results: For studying cervical cancer, 40 cases and 40 matched controls were interviewed. In the univariable and multivariable analyses older age, depression or anxiety and ever smoking seemed to act as independent risk factors/markers, while older age at first intercourse was protective. For studying CIN3, 99 cases and 79 controls were interviewed. Multivariable analysis has demonstrated that being born in Israel, depression or anxiety and ever smoking were independent risk factors/markers for CIN3. Conclusions: The risk factors/markers studied, that were associated with cervical cancer or CIN3 among Jewish women in Israel, are similar to those reported in other parts of the world, and do not explain the observed discrepancy of high in-situ cervical cancer rates and low invasive cervical cancer incidence in Israel.
\end{abstract}

Keywords: Cervical cancer - CIN3 - risk factors - case control studies - Israel

Asian Pac J Cancer Prev, 17 (4), 2067-2073

\section{Introduction}

In 2012, invasive cervical cancer was rated fourth among women in the world, with an age-adjusted incidence rate of 14.0 per 100,000 (WHO, 2013). Cervical Intraepithelial Neoplasia 3 (CIN3) is a local in-situ transformation, which represents with abnormal growth of squamous cells on the surface of the cervix and is on the natural continuum leading from a Human Papillomavirus (HPV) infection to cervical cancer. According to the Israel National Cancer Registry, in 2012 the age-adjusted incidence rate of cervical cancer among Jewish women was low $(5.1$ per 100,000$)$ while that of CIN3 was relatively high, 24.5 per 100,000 (ICDC, 2015).

Risk factors for cervical diseases are divided into two major groups: proven and possible. One of the wellaccepted proven risk factor for cervical morbidity is HPV infection. The causal relationship between HPV infection and cervical cancer has been proven beyond doubt (Kirwan et al., 2001; Bosch et al., 2002). Walboomers and colleagues (Walboomers et al., 1999) have shown in 1999 that $99.7 \%$ of women with cervical cancer were HPV-positive. They were also able to demonstrate that HPV attributable fraction in cancer incidence among humans is the highest detected (Walboomers et al., 1999). Yet, HPV was defined as a risk factor which is necessary but not exclusive nor sufficient for the development of cervical cancer (Giuntoli et al., 2008). In addition, age is another proven risk factor, since cervical cancer occurs mainly in middle life or later, with an average age of diagnosis at 52 years. However, there are two main incidence peaks: in the age groups 35-39 and 60-64 years (Cox, 2006; Giuntoli et al., 2008). Smoking is also a proven independent risk factor for cervical cancer through genotoxic and immunosuppressive mechanisms (Giuntoli et al., 2008). Possible risk factors for cervical morbidity includes: low socio-economic status, having sexually transmitted diseases, hormonal factors (such as number of pregnancies, intake of ovulation-inducing agents, exposure to DiEthylStilbestrol, etc.), low compliance

${ }^{1}$ Department of Epidemiology and Preventive Medicine, School of Public Health, Sackler Faculty of Medicine, ${ }^{5}$ Stanley Steyer Institute for Epidemiology and Research, Tel Aviv University, ${ }^{3}$ Women Health Center, Maccabi Healthcare Services, Tel Aviv, ${ }^{2}$ Israel Center for Disease Control, Ministry of Health, Sheba Medical Center, Ramat Gan, ${ }^{4}$ Department of Gynecologic Oncology, Sheba Medical Center, Tel Hashomer, ${ }^{6}$ School of Public Health, University of Haifa, Haifa, Israel *For correspondence: rbassal@gmail.com 
to Pap test, younger age at first intercourse, having sex with multiple partners and medical conditions such as immunosuppression (Sillman et al., 1984; Bornstein et al., 1995; Ngelangel et al., 1998; Giuntoli et al., 2008). Possible protective factor for cervical morbidity includes high consumption of fruits and vegetables and other dietary factors (Bornstein et al., 1995; García-Closas et al., 2005).

Israel does not have a national cervical cancer screening program, and pap testing is opportunistic and selective. Hence incidence rates in Israel are expected to reflect the natural disease course, as is the case in other countries, where higher incidence of pre-malignant and in-situ tumors is in correlation with the rates of invasive carcinomas. In light of the discrepancy of the relatively high incidence of CIN3 and the relatively low incidence of invasive cervical cancer in Israel, the aim of this study was to explore risk and protective factors associated with cervical cancer and CIN3 among Israeli women, in order to identify unique exposures that may partially explain this picture.

\section{Materials and Methods}

Two case control studies based on prevalent cases (due to the rarity of the disease) were conducted to detect risk factors/markers, the first focusing on invasive cervical cancer and the second, on CIN3.

\section{Invasive Cervical Cancer Matched Case-Control Study}

Invasive cervical cancer cases were obtained from the following clinics: a. Sheba Medical Center (SMC), a tertiary hospital in the center of Israel with a turnover of around 40 new cervical cancer cases each year. The study was presented to patients who attended the oncogynecology clinic, after diagnosis and initial treatment. The patients were requested by their medical doctors to participate in the study after detailed explanation of its importance and women who agreed, signed an informed consent form and received a self-administered structured questionnaire. b. Maccabi Healthcare Services (MHS), the second largest Health Maintenance Organization (HMO) in Israel, covering approximately $30 \%$ of the total population. Women diagnosed with invasive cervical cancer were asked to participate in the study by a specialized gynecologist. After obtaining an oral agreement, the questionnaire was mailed to the women with a prepaid stamped return envelope.

Controls were defined as healthy women, who reported not having an abnormal Pap test in the last 15 years, and who attended their MHS gynecologist for a periodic examination. To avoid differences between cases and controls in sexual behavior expected between different regions in Israel, controls were matched to cases by residency.

Sample size was calculated based on the expected smoking proportions among cases and controls. For the following indices: $\alpha=0.05$, power $(1-\beta)=0.8$, smoking proportion of 0.5 among cases and 0.2 among controls, 2 sided tests, the sample size calculated was 39 in each group.
Response rate: In SMC, 50 patients were treated for invasive cervical cancer between November 2011 and January 2013 (personal communication), of whom 18 agreed to participate (response rate $36.0 \%$ ). In MHS, the number of women diagnosed with invasive cervical cancer between 2008 and 2010 was 119, of them, 9 died, 35 agreed to participate but only 22 have sent the questionnaire back (response rate $20.0 \%$ ). Totally, 40 cases participated (total response rate: $25.0 \%$ ). Between July 2010 and December 2012, 79 controls agreed to participate, representing $30 \%$ of the women who were asked to participate in the study, according to their gynecologist. Of them, 40 controls were matched to the cases by address (selecting the control living in the immediate vicinity of the case).

Statistical Analysis: Differences in proportions between cases and controls were assessed using the McNemar's test and for continuous variables, using the paired t-test. Conditional logistic regression models were used for the univariable and multivariable analyses. Matched odds ratios (MORs) and 95\% confidence intervals (95\% CI) were calculated for all variables. Multivariable analysis included variables which were significantly associated with cervical morbidity in the univariable analysis. Effect modification and interaction were assessed for each covariate associated with morbidity.

\section{CIN3 Case-Control Study}

CIN3 cases were obtained from MHS clinic in central Israel (Tel-Aviv city) by a specialized gynecologist, who contacted patients that underwent conization due to CIN3 in his clinic and asked them to participate. Women who consented to participate received a self-administered structured questionnaire.

Controls were defined as healthy women, who reported not having an abnormal Pap test in the last 15 years, and who attended their MHS gynecologist for a periodic examination. The control participants were not matched to the cases.

Sample size was calculated based on the expected smoking proportions among cases and controls. For the following indices:, $\alpha=0.05$, power $(1-\beta)=0.8$, a smoking proportion of 0.4 among cases and 0.2 among controls, 2 sided tests, the sample size calculated was 82 in each group.

Response rate: Between June 2010 and June 2013, 255 CIN3 cases were treated due to CIN3 in the MHS clinic in Tel Aviv, of whom 99 agreed to participate and the total response rate was $38.8 \%$. Between July 2010 and December 2012, 79 control women agreed to participate, representing $30 \%$ of the women requested to participate in the study by their gynecologist.

Statistical Analysis: Differences in proportions between cases and controls were assessed using the chi-square test and for continuous variables, using the student t-test. Logistic regression models were used for the univariable and multivariable analyses. Odds ratios (ORs) and (95\% CI were calculated for all variables. Multivariable analysis included variables which were significantly associated with cervical morbidity in the univariable analysis. Effect modification and interaction were assessed for each covariate associated with morbidity. 
Risk Factors for Cervical Cancer and CIN3 in Jewish Women in Israel -Two Case Control Studies

Table 1. Demographic Characteristics of Cases (Cervical Cancer and CIN3) and Controls

\begin{tabular}{|c|c|c|c|c|c|}
\hline & & \multicolumn{2}{|c|}{ Cervical Cancer } & \multicolumn{2}{|c|}{ CIN3 } \\
\hline & & Cases $(\mathrm{N}=40)$ & Controls $(\mathrm{N}=40)$ & Cases $(\mathrm{N}=99)$ & Controls $(\mathrm{N}=79)$ \\
\hline \multirow{2}{*}{ Age } & $\begin{array}{c}\text { Age (mean } \pm \text { Standard } \\
\text { Error })\end{array}$ & $44.5 \pm 1.8$ & $36.1 \pm 1.6$ & $34.0 \pm 0.7$ & $34.4 \pm 1.1$ \\
\hline & $\begin{array}{c}\text { Age at immigration } \\
\text { (mean } \pm \text { Standard Error) }\end{array}$ & $30.4 \pm 3.2$ & $15.1 \pm 2.5$ & $12.1 \pm 2.0$ & $15.0 \pm 1.8$ \\
\hline \multirow{2}{*}{ Birth country } & Israel & $23 / 40(57.5)$ & $25 / 40(62.5)$ & $81 / 99(81.8)$ & 49/79 (62.0) \\
\hline & Other & $17 / 40(42.5)$ & $15 / 40(37.5)$ & $18 / 99(18.2)$ & $30 / 79(38.0)$ \\
\hline \multirow{2}{*}{ Religion } & Jewish & $32 / 40(80.0)$ & 40/40 (100.0) & 97/99 (98.0) & 78/79 (98.7) \\
\hline & Other & $8 / 40(20.0)$ & $0 / 40(0.0)$ & 2/99 (2.0) & 1/79 (1.3) \\
\hline \multirow[b]{2}{*}{ Marital status } & Single & $4 / 40(10.0)$ & $11 / 40(27.5)$ & 27/98 (27.6) & 29/79 (36.7) \\
\hline & $\begin{array}{c}\text { Other (married/widow/ } \\
\text { divorcee) }\end{array}$ & $36 / 40(90.0)$ & $29 / 40(72.5)$ & $71 / 98(72.4)$ & $50 / 79(63.3)$ \\
\hline \multirow[t]{2}{*}{ Education } & $\begin{array}{c}\text { High school diploma } \\
\text { or less }\end{array}$ & 23/39 (59.0) & $17 / 39(43.6)$ & 37/97 (38.1) & $33 / 76(43.4)$ \\
\hline & Academic degree & $16 / 39(41.0)$ & $22 / 39(56.4)$ & 60/97 (61.9) & 43/76 (56.6) \\
\hline \multirow[b]{2}{*}{ Income } & Beyond average $^{\wedge}$ & $16 / 38(42.1)$ & $9 / 36(25.0)$ & $11 / 90(12.2)$ & $15 / 69(21.7)$ \\
\hline & $\begin{array}{c}\text { Equal or among } \\
\text { average }^{\wedge}\end{array}$ & $22 / 38(57.9)$ & $27 / 36(75.0)$ & $79 / 90(87.8)$ & $54 / 69(78.3)$ \\
\hline
\end{tabular}

${ }^{\wedge}$ The average income in Israeli in 8,500 New Israeli Shekels

Table 2. Univariate and Multivariable Conditional Logistic Regression Analyses for Variables Predicting Cervical Cancer

\begin{tabular}{|c|c|c|c|c|c|}
\hline Univariate analysis & Cases n/N (\%) & Controls n/N (\%) & $\mathrm{MOR}^{*}$ & $95 \% \mathrm{CI}$ & P value \\
\hline Age (Mean \pm S.E.) & $44.5 \pm 1.8$ & $36.1 \pm 1.6$ & 1.1 & $1.0-1.2$ & $<0.01$ \\
\hline Birth country (Israel vs. Other)* & $23 / 40(57.5)$ & $25 / 40(62.5)$ & 1.7 & $0.5-5.6$ & 0.42 \\
\hline Income (<Average vs. $\geq$ Average $)^{*}$ & $16 / 38(42.1)$ & $9 / 36(25.0)$ & 4.3 & $0.8-22.2$ & 0.08 \\
\hline $\begin{array}{l}\text { Performing screening test (mammography or } \\
\text { pap) routinely (Yes vs. No)* }\end{array}$ & $27 / 40(67.5)$ & $26 / 40(65.0)$ & 0.3 & $0.1-1.4$ & 0.13 \\
\hline Age at first intercourse $(\text { Mean } \pm \text { S.E. })^{*}$ & $18.2 \pm 0.3$ & $19.2 \pm 0.6$ & 0.6 & $0.4-0.9$ & 0.02 \\
\hline $\begin{array}{l}\text { Number of sex partners during life ( }>5 \text { vs. } \\
\leq 5)^{*}\end{array}$ & $18 / 40(45.0)$ & $11 / 40(27.5)$ & 2.7 & $0.9-8.3$ & 0.08 \\
\hline $\begin{array}{l}\text { Cervical cancer or CIN3 in a family member } \\
\text { (Yes vs. No)* }\end{array}$ & $4 / 40(10.0)$ & $4 / 40(10.0)$ & 0.7 & $0.1-8.0$ & 0.74 \\
\hline Physical illness (Yes vs. No)* & $27 / 40(67.5)$ & $23 / 40(57.5)$ & 1.4 & $0.4-4.9$ & 0.57 \\
\hline Depression or anxiety (Yes vs. No)* & $10 / 40(25.0)$ & $2 / 40(5.0)$ & 11.6 & $1.1-120.8$ & 0.04 \\
\hline Sexual transmitted diseases (Yes vs. No)* & $11 / 40(27.5)$ & $5 / 40(12.5)$ & 1.8 & $0.4-7.7$ & 0.4 \\
\hline Consumption of medications (Yes vs. No)* & $25 / 40(62.5)$ & $20 / 40(50.0)$ & 2.2 & $0.7-6.7$ & 0.17 \\
\hline $\begin{array}{l}\text { Consumption of birth control pills (Yes vs. } \\
\text { No)* }\end{array}$ & $23 / 40(57.5)$ & $28 / 40(70.0)$ & 1.4 & $0.4-4.7$ & 0.6 \\
\hline Contraceptive use (Yes vs. No)* & $29 / 39(74.4)$ & $24 / 40(60.0)$ & 2.4 & $0.7-8.8$ & 0.17 \\
\hline Smoking ever (Yes vs. No)* & $29 / 40(72.5)$ & $16 / 40(40.0)$ & 4.2 & $1.3-14.0$ & 0.02 \\
\hline Dietary supplements (Yes vs. No)* & $27 / 40(67.5)$ & $29 / 40(72.5)$ & 0.4 & $0.1-1.4$ & 0.14 \\
\hline Healthy lifestyle (Yes vs. No)* & $30 / 40(75.0)$ & $28 / 40(70.0)$ & 1.1 & $0.4-3.7$ & 0.83 \\
\hline High risk sex (Yes vs. No)* & $26 / 40(65.0)$ & $28 / 40(70.0)$ & 1.4 & $0.4-4.6$ & 0.54 \\
\hline Number of pregnancies (Mean \pm S.E.)* & $3.3 \pm 0.5$ & $1.8 \pm 0.3$ & 1.2 & $0.9-1.6$ & 0.12 \\
\hline \multicolumn{6}{|l|}{ Multivariable analyses } \\
\hline \multicolumn{6}{|l|}{ Model 1} \\
\hline Age & & & 1.3 & $1.1-1.6$ & 0.01 \\
\hline Age at first intercourse & & & 0.4 & $0.2-0.9$ & 0.02 \\
\hline Depression or anxiety & & & 90.8 & $2.1-4016.1$ & 0.02 \\
\hline Smoking ever & & & 2.4 & $0.3-17.2$ & 0.38 \\
\hline \multicolumn{6}{|l|}{ Model 2} \\
\hline Age & & & 1.2 & $1.1-1.3$ & $<0.01$ \\
\hline Age at first intercourse & & & 0.7 & $0.5-1.0$ & 0.08 \\
\hline Smoking ever & & & 2.6 & $0.7-9.3$ & 0.15 \\
\hline \multicolumn{6}{|l|}{ Model 3} \\
\hline Age & & & 1.2 & $1.0-1.3$ & $<0.01$ \\
\hline Depression or anxiety & & & 17.8 & $1.2-271.3$ & 0.04 \\
\hline Smoking ever & & & 5.5 & $1.2-25.8$ & 0.03 \\
\hline
\end{tabular}

*Age adjusted; S.E. - Standard Error 
The questionnaire retrieved data on screening tests performance (Pap or mammogram), age at first intercourse, number of sex partners, family history of cervical diseases, physical illness (headaches, allergy, asthma, diabetes, immunodeficiency, autoimmune diseases, cancer, any other chronic disease), depression or anxiety, sexually transmitted diseases, consumption of medications (any medication aimed at treating asthma and allergy, non-steroidal anti-inflammatory agents, immune system depressants, di-ethyl stilbestrol, antidepressants, any other chronic medication), contraceptive use, smoking habits, consumption of food supplements, health lifestyle (balanced diet and exercising), sex habits, number of pregnancies and demographic variables (age, country of birth, income, etc.). The questionnaire was self-reported, self-administered and anonymous because of its intrusiveness.

The study obtained the approval of the Ethics Committee of the SMC and the MHS.

Data analysis was performed using SAS software release 9.1.3 (SAS Institute Inc., USA). For all statistical tests, two-sided p-value of $<0.05$ was considered statistically significant.

\section{Results}

Table 1 presents the demographic characteristics of cervical cancer and CIN3 cases and their corresponding controls. The mean age of the 40 invasive cervical cancer cases was 44.5 years and that of the 40 matched controls was 36.1 yaears. The mean age of the 99 CIN3 cases was 34.0 years while that of the 79 non-matched controls was 34.4 years. Most patients were Jewish $(80.0 \%$ of cervical cancer and $98.0 \%$ of CIN3 cases).

Table 2 presents the univariable and multivariable conditional logistic regression analyses for variables associated with cervical cancer. The univariable analysis traced a significant association with the following risk factors/markers: age (Matched Odds Ratio $(\mathrm{MOR})=1.1, \mathrm{p}$-value $<0.01)$, depression or anxiety $(\mathrm{MOR}=11.6, \mathrm{p}$-value $=0.04)$ and ever smoking $(\mathrm{MOR}=4.2$, $\mathrm{p}$-value $=0.02)$. Older age at first intercourse $(\mathrm{MOR}=0.6$, $\mathrm{p}$-value $=0.02$ ) was found to be a protective factor. Interaction and effect modification were not detected. In the multivariable analyses, these variables continued to be significantly associated with cervical cancer in different models.

Table 3 presents the univariable and multivariable logistic regression analyses for CIN3. The univariable analysis traced significant associations with the following risk factors/markers: being born in Israel (vs. being born in other countries $)(\mathrm{OR}=2.8, \mathrm{p}$-value $<0.01)$, number of $\mathrm{sex}$ partners during life ( $>5$ vs. $\leq 5)(\mathrm{OR}=1.9$, p-value $=0.04)$, depression or anxiety $(\mathrm{OR}=3.9, \mathrm{p}$-value $=0.02)$ and ever smoking $(\mathrm{OR}=2.4, \mathrm{p}$-value $<0.01)$. Interaction and effect modification were not detected. In the multivariable analysis, being born in Israel (vs. being born in other countries $)(\mathrm{OR}=2.6$, p-value $=0.01)$, depression or anxiety $(\mathrm{OR}=4.4, \mathrm{p}$-value $=0.02)$ and ever smoking $(\mathrm{OR}=2.1$, $\mathrm{p}$-value $=0.02)$ kept their significant association with CIN3.

Table 3. Univariable and Multivariable Logistic Regression Analyses for Variables Predicting CIN3

\begin{tabular}{|c|c|c|c|c|c|}
\hline & Cases n/N (\%) & Controls $\mathrm{n} / \mathrm{N}(\%)$ & OR & $95 \% \mathrm{CI}$ & $P$ value \\
\hline \multicolumn{6}{|l|}{ Univariable analysis } \\
\hline Age (Mean+S.E.) & $34.0 \pm 0.7$ & $34.4 \pm 1.1$ & 1.0 & $0.9-1.1$ & 0.73 \\
\hline Birth country (Israel vs. Other) & $81 / 99(81.8)$ & $49 / 79(62.0)$ & 2.8 & $1.4-5.5$ & $<0.01$ \\
\hline Income (<Average vs. $\geq$ Average) & $11 / 90(12.2)$ & $15 / 69(21.7)$ & 0.5 & $0.2-1.2$ & 0.11 \\
\hline $\begin{array}{l}\text { Performing screening test (mammography or pap) } \\
\text { routinely (Yes vs. No) }\end{array}$ & $68 / 99(68.7)$ & $52 / 79(65.8)$ & 1.1 & $0.6-2.1$ & 0.69 \\
\hline Age at first intercourse (Mean \pm S.E.) & $18.3 \pm 0.2$ & $18.9 \pm 0.4$ & 0.9 & $0.8-1.0$ & 0.13 \\
\hline Number of sex partners during life (>5 vs. $\leq 5$ ) & $53 / 99(53.5)$ & $30 / 79(38.0)$ & 1.9 & $1.0-3.4$ & 0.04 \\
\hline Cervical cancer or CIN3 in a family member (Yes vs. No) & $12 / 99(12.1)$ & $3 / 79(3.8)$ & 3.5 & $0.9-12.8$ & 0.06 \\
\hline Physical illness (Yes vs. No) & $55 / 99(55.6)$ & $46 / 79(58.2)$ & 0.9 & $0.5-1.6$ & 0.72 \\
\hline Depression or anxiety (Yes vs. No) & $17 / 99(17.2)$ & $4 / 79(5.1)$ & 3.9 & $1.3-12.1$ & 0.02 \\
\hline Sexual transmitted diseases (Yes vs. No) & $21 / 99(21.2)$ & $14 / 79(17.7)$ & 1.2 & $0.6-2.7$ & 0.56 \\
\hline Consumption of medications (Yes vs. No) & $46 / 99(46.5)$ & $39 / 79(49.4)$ & 0.9 & $0.5-1.6$ & 0.7 \\
\hline Consumption of birth control pills (Yes vs. No) & $88 / 99(88.9)$ & $62 / 79(78.5)$ & 2.2 & $0.9-5.0$ & 0.06 \\
\hline Contraceptive use (Yes vs. No) & 73/99 (73.7) & $56 / 79(70.9)$ & 1.2 & $0.6-2.2$ & 0.67 \\
\hline Smoking ever (Yes vs. No) & $67 / 99(67.7)$ & $37 / 79(46.8)$ & 2.4 & $1.3-4.4$ & $<0.01$ \\
\hline Dietary supplements (Yes vs. No) & $65 / 99(65.7)$ & $56 / 79(70.9)$ & 0.8 & $0.4-1.5$ & 0.46 \\
\hline Healthy lifestyle (Yes vs. No) & 78/99 (78.8) & $60 / 79(76.0)$ & 1.2 & $0.6-2.4$ & 0.65 \\
\hline High risk sex (Yes vs. No) & $86 / 99(86.9)$ & $63 / 79(79.8)$ & 1.7 & $0.8-3.7$ & 0.2 \\
\hline Number of pregnancies (Mean \pm S.E.) & $1.7 \pm 0.2$ & $1.5 \pm 0.2$ & 1.0 & $0.9-1.2$ & 0.6 \\
\hline \multicolumn{6}{|l|}{ Multivariable analysis } \\
\hline Birth Country (Israel vs. Other) & & & 2.6 & $1.3-5.3$ & 0.01 \\
\hline Number of sex partners during life ( $>5$ vs. $\leq 5$ ) & & & 1.5 & $0.8-2.9$ & 0.23 \\
\hline Depression or anxiety & & & 4.4 & $1.3-13.5$ & 0.02 \\
\hline Smoking ever & & & 2.1 & $1.1-4.1$ & 0.02 \\
\hline
\end{tabular}

S.E. - Standard Error 


\section{Discussion}

The aim of this study was to identify risk and protective factors/markers for invasive cervical cancer and CIN3 in Israel in order to settle the conflict between the relatively high rates of pre-malignant morbidity and the relatively low rates of invasive tumors in the Jewish population.

Ever smoking was found to be a risk factor for cervical cancer and for CIN3. Other studies have also demonstrated an association between smoking and cervical disease (Trevathan et al., 1983; Castle et al., 2002). In a cohort study performed in Portland, Oregon, among 1,812 women positive for oncogenic-type HPV with a follow up that lasted 10 years, the relative risk (RR) for cervical cancer and CIN3 (combined) among smokers compared with non-smokers was 3.3 (95\% CI: 1.6-6.7) (Castle et al., 2002). A case-control study conducted among 374 cases and 266 controls in the age group of 17-55 years in Atlanta, Georgia, found that smoking was associated with in-situ cervical cancer, i.e., CIN3 (rate ratio $=3.6,95 \% \mathrm{CI}$ : 2.1-6.2), with severe dysplasia (rate ratio $=3.3,95 \%$ CI: 1.9 5.8 ) and with mild to moderate dysplasia (rate ratio $=2.4$, 95\%CI: 1.6-3.7) (Trevathan et al., 1983). Previous study from the former Soviet Union concluded that smoking in itself is not an independent risk factor for CIN2+, but is mediated by the presence of high risk HPV (Syrjänen et al., 2007). However, a study from North Sweden had demonstrated that smoking is independently associated with CIN2-3, even after adjustment to HPV (Kjellberg et al., 2000). Possible explanations for the association found between smoking and cervical morbidity are: (a) Damage to the local immune system - a decrease in the number of Langerhans cells in the cervix epithelium, which has demonstrated an inverse dose-response association with the number of cigarettes smoked (Barton et al., 1988). The Langerhans cells play an important role in the immune system: after identifying an antigen, these cells migrate towards the closest lymph node, present the antigen and promote the creation of memory cells (Barton et al., 1988). (b) Direct carcinogenic effects - the carcinogenic nitrosamine 4-(methylnitrosamino)-1- (3-pyridyl)-1butanone (NNK) found in cigarettes was detected in the cervical mucus of women who smoke cigarettes (Prokopczyk et al., 1997). Cotinine and nicotine were also present in cervical mucus of cigarette smokers (Bornstein et al., 1995). These compounds are related to a decline in the local protective immune mechanisms (Bornstein et al., 1995).

Depression or anxiety were found in the current study to be risk markers for cervical cancer and for CIN3. Additional studies have demonstrated an association between cervical morbidity and anxiety and stress (Coker et al., 2003; Pereira et al., 2003; Wang et al., 2005). The ASCUS LSIL Triage Study (ALTS) conducted on women positive for oncogenic-type HPV found high risk for having CIN2 among women who reported higher levels of depression (OR=1.9,95\%CI: 1.1-3.1) (Wang et al., 2005). A study conducted in South Carolina among low income women found that stress plays a role in the development of precancerous lesions (Coker et al., 2003). A study conducted among 32 African-American and
Caribbean-American women positive for HPV and HIV has shown that psychological stress is a risk marker for the development of precancerous lesions $(\mathrm{OR}=8.6,95 \% \mathrm{CI}$ : 1.1-69.1) (Pereira et al., 2003). Possible explanations for this finding are: (a) Depression or anxiety and medical therapy for depression and anxiety may have a negative impact on the immune system thus impairing its ability to eliminate HPV from the body after infection or from the formed lesion at a later stage. (b) Depression or anxiety may be related to other risk factors that influence exposure to HPV, such as smoking and having sex with multiple partners. Thus, depression or anxiety may be a mediator and not necessarily a direct risk marker. (c) Depressed women are less likely to perform routine Pap smears (Vigod et al., 2001) and may be diagnosed only after the onset of symptoms. (d) Reversed causality: Invasive cervical cancer cases were asked to fill out the questionnaire after initial treatment while CIN3 patients were asked to do so just before their surgery. Therefore the findings may be attributed to the anxiety the patients were in because of their physical condition. The question whether depression or anxiety precede cervical disease or are caused by cervical morbidity is controversial; a study from Canada that explored this issue found more psychological disorders among women undergoing colposcopy assessment, i.e. before they knew their final diagnosis (Le et al., 2006). Thus there is high likelihood that the explanation to our finding is not circumstantial, and in depth study will provide further insight to this important issue.

Being born in Israel was found in this study to be a risk marker for developing CIN3. This finding may be explained by the fact that women born in Israel may have a more permissive sexual behavior in comparison to those not born in Israel, raising the comprehensive sex education issue. Another explanation is the awareness of Israel-born women to Pap test, which increases their chances of being diagnosed.

Having sex with more than five partners was found to be positively associated with CIN3 in the current study. A similar association was reported by a study conducted in the United Kingdom, that found that the risk for Cervical Squamous Cell Carcinoma and Adenocarcinoma was higher among women who had sexual relations with 5+ partners, compared to only one (Green et al., 2003). This finding may be explained by the increased chance of acquiring sexually-transmitted HPV infection.

Older age was found in our study to be a risk marker for cervical cancer. This issue was already discussed in detail by others (Cox, 2006; Giuntoli et al., 2008) and may be explained by: (a) There is long latency period between the exposure to HPV and the diagnosis of CIN3 and cervical cancer. (b) Advancing age is often accompanied by a decrease in the immune system function and in its ability to cope with abnormal growth of cells. (c) Advanced age is associated with higher likelihood of ever being exposed to certain risk factors, including HPV, and a cumulative effect of these factors may occur.

We found an inverse association between age at first intercourse and the risk for invasive cervical cancer, an observation previously reported by others (Ngelangel 
et al., 1998; Deacon et al., 2000). A case-control study nested in the Manchester cohort found that having sex for the first time at the age of $>21$ years was associated with lower risk of acquiring $\mathrm{CIN} 3$ ( $\mathrm{OR}=0.3$, 95\% CI: 0.1-0.8) (Deacon et al., 2000). A case-control study conducted in the Philippines found that women whose first sexual intercourse happened at the age of $<18$ years, had higher chance for a Cervical Squamous Cell Carcinoma $(\mathrm{OR}=5.0$, 95\% CI: 3.1-7.9), after adjustment for age (Ngelangel et al., 1998). Women experiencing their first sexual relationship at an older age are probably less exposed to HPV.

The main advantage of the study is the relatively large number of CIN3 cases and the variety of risk and protective factors/markers for cervical malignancy that was examined.

However, the following limitations should be kept in mind: (a) Low response rate. For cervical cancer, the response rate was $25 \%$, for CIN3, 39\% and for the controls, $30 \%$. Other studies reported higher response rates (Hoyo et al., 2000; Shields et al., 2004). The low response rates may be explained by the fact that patients were mostly from outpatients clinics and from an HMO, and their low response rates reflect those of healthy people (CDC, 2008). The low response rate among cases and controls may also be explained by the intricacy of the questionnaire. (b) We had no data on HPV infection history among the patients. (c) Data on non-respondents was not available and we could not compare the characteristics of those who participated to the characteristics of those who declined participation. (d) The study heavily relied on personal report. (e) Recall bias - in case-control study, cases are more likely to remember exposures differently than the controls, especially exposures that are commonly perceived as associated with the outcome.

In conclusion, our study does not satisfactorily resolve the discrepancy of the high incidence of CIN3 and the low incidence of invasive cervical cancer in Israeli Jewish women, primarily hypothesized to be associated with differences in the distribution of certain risk factors. The discrepancy, however, cannot be explained by the opportunistic screening policy in Israel, which unfortunately covers only around $40 \%$ of the target population and is usually less accessible to those at the highest risk for cervical cancer. Since we have previously demonstrated that the distribution of HPV genotypes among cervical cancer and CIN3 cases in Israel is similar to that reported from other countries in the world (Bassal et al., 2015), different distribution of HPV types in Israeli women cannot serve as a potential explanation either. However, another hypothesis that should be taken into consideration, and which was not yet tested in Israel, is the differences in the HPV genome variants, as was demonstrated by Freitas et al. (2014) or the polymorphism of 553 proteins in Jewish population, as was demonstrated by Arbel-Alon et al. (2002), so further research is warranted.

\section{References}

Arbel-Alon S, Menczer J, Feldman N, et al (2002) Codon 72 polymorphism of p53 in Israeli Jewish cervical cancer patients and healthy women. Int J Gynecol Cancer, 12, 741-4.

Barton SE, Maddox PH, Jenkins D, et al (1988) Effect of cigarette smoking on cervical epithelial immunity: a mechanism for neoplastic change? Lancet, 2, 652-4.

Bassal R, Rosin LS, Schvimer M, et al (2015) Prevalence and correlates of human papillomavirus genotypes among patients with cervical cancer and cervical intraepithelial neoplasia 3 in Israel. J Low Genit Tract Dis, 19, 161-4.

Bornstein J, Rahat MA, Abramovici H (1995) Etiology of cervical cancer: current concepts. Obstet Gynecol Surv, 50, 146-54.

Bosch FX, Lorincz A, Muñoz N, Meijer CJ, Shah, KV (2002) The causal relation between human papillomavirus and cervical cancer. J Clin Pathol, 55, 244-65.

Castle PE, Wacholder S, Lorincz AT, et al (2002) A prospective study of high-grade cervical neoplasia risk among human papillomavirus-infected women. J Natl Cancer Inst, 94, 1406-14.

Centers for Disease Control and Prevention (CDC) (2008) Behavioral Risk Factor Surveillance System: 2005 Summary Data Quality Report.

Coker AL, Bond S, Madeleine MM, Luchok K, Pirisi L (2003) Psychosocial stress and cervical neoplasia risk. Psychosom Med, 65, 644-51.

Cox JT (2006) Epidemiology and natural history of HPV. $J$ Fam Pract, 3-9.

Deacon JM, Evans CD, Yule R, et al (2000) Sexual behaviour and smoking as determinants of cervical HPV infection and of CIN3 among those infected: a case-control study nested within the Manchester cohort. Br J Cancer, 83, 1565-72.

Freitas LB, Chen Z, Muqui EF, et al (2014) Human papillomavirus 16 non-European variants are preferentially associated with high-grade cervical lesions. PLoS One, 9, e100746.

García-Closas R, Castellsagué X, Bosch X, González CA (2005) The role of diet and nutrition in cervical carcinogenesis: a review of recent evidence. Int J Cancer, 117, 629-37.

Giuntoli LR, Bristow RE (2008) Chapter 58: cervical cancer. In: Gibbs RS, Karlan BY, Haney AF, et al, editors. Danforth's obstetrics and gynecology, $10^{\text {th }}$ ed. Philadelphia, PA: Lippincott Williams \& Wilkins, 971-88.

Green J, Berrington de Gonzalez A, Sweetland S, et al (2003) Risk factors for adenocarcinoma and squamous cell carcinoma of the cervix in women aged 20-44 years: the UK national case-control study of cervical cancer. $\mathrm{Br} \mathrm{J}$ Cancer, 89, 2078-86.

Hoyo C, Miller WC, Newman BM, Fortney JA (2000) Selective screening for cervical neoplasia: an approach for resourcepoor settings. Int J Epidemiol, 29, 807-12.

Israeli Center for Disease Control (ICDC), Israeli National Cancer Registry, Ministry of Health (2015) Annual Reports. 1980-2012. http://www.health.gov.il/UNITSOFFICE/HD/ ICDC/ICR/CANCERINCIDENCE/Pages/about.aspx

Kirwan JM, Herrington CS (2001) Human papillomavirus and cervical cancer: where are we now? BJOG, 108, 1204-13.

Kjellberg L, Hallmans G, Ahren AM, et al (2000) Smoking, diet, pregnancy and oral contraceptive use as risk factors for cervical intra-epithelial neoplasia in relation to human papillomavirus infection. Br J Cancer, 82, 1332-8.

Le T, Hopkins L, Menard C, et al (2006) Psychologic morbidities prior to loop electrosurgical excision procedure in the treatment of cervical intraepithelial neoplasia. Int J Gynecol Cancer, 16, 1089-93.

Ngelangel C, Munoz N, Bosch FX, et al (1998) Causes of cervical cancer in the Philippines: a case-control study. $J$ Natl Cancer Inst, 90, 43-9.

Pereira DB, Antoni MH, Danielson A, et al (2003) Life stress 
and cervical squamous intraepithelial lesions in women with human papillomavirus and human immunodeficiency virus. Psychosom Med, 65, 427-34.

Prokopczyk B, Cox JE, Hoffmann D, Waggoner SE (1997) Identification of tobacco-specific carcinogen in the cervical mucus of smokers and nonsmokers. J Natl Cancer Inst, 89, 868-73.

Shields TS, Brinton LA, Burk RD, et al (2004) A case-control study of risk factors for invasive cervical cancer among U.S. women exposed to oncogenic types of human papillomavirus. Cancer Epidemiol Biomarkers Prev, 13, 1574-82.

Sillman F, Stanek A, Sedlis A, et al (1984) The relationship between human papillomavirus and lower genital intraepithelial neoplasia in immunosuppressed women. $A m$ J Obstet Gynecol, 150, 300-8.

Syrjänen K, Shabalova I, Petrovichev N, et al (2007) Smoking is an independent risk factor for oncogenic human papillomavirus (HPV) infections but not for high-grade CIN. Eur J Epidemiol, 22, 723-35.

Trevathan E, Layde P, Webster LA, et al (1983) Cigarette smoking and dysplasia and carcinoma in situ of the uterine cervix. JAMA, 250, 499-502.

Vigod SN, Kurdyak PA, Stewart DE, Gnam WH, Goering PN (2001) Depressive symptoms as a determinant of breast and cervical cancer screening in women: a population-based study in Ontario, Canada. Arch Womens Ment Health, 14, 159-68.

Walboomers JM, Jacobs MV, Manos MM, et al (1999) Human papillomavirus is a necessary cause of invasive cervical cancer worldwide. J Pathol, 189, 12-9.

Wang SS, Schiffman M (2005) Medication use, medical conditions, and the risk of human papillomavirus infection and subsequent cervical intraepithelial neoplasia 3 among women with mild cytologic abnormalities. Cancer Epidemiol Biomarkers Prev, 14, 542-5.

World Health Organization (WHO) (2013). GLOBOCAN 2012. Cervical cancer incidence and mortality worldwide in 2012 summary. Cancer fact sheet. (http://globocan.iarc.fr/Pages/ fact_sheets_population.aspx) 